\title{
Periodic solutions of Rayleigh equations with singularities
}

\author{
Zaihong Wang ${ }^{1 *}$ and Tiantian $\mathrm{Ma}^{2}$
}

\author{
"Correspondence: \\ zhwang@cnu.edu.cn \\ 'School of Mathematical Sciences, \\ Capital Normal University, Beijing, \\ 100048, People's Republic of China \\ Full list of author information is \\ available at the end of the article
}

\begin{abstract}
In this paper, we study the existence of periodic solutions of Rayleigh equations with singularities $x^{\prime \prime}+f\left(t, x^{\prime}\right)+g(x)=p(t)$. By using the limit properties of the time map, we prove that the given equation has at least one $2 \pi$ periodic solution.
\end{abstract}

MSC: $34 C 11 ; 34 C 15 ; 34 C 25$

Keywords: Rayleigh equation; singularity; periodic solution; continuation lemma; time map

\section{Introduction}

In this paper, we are concerned with the existence of periodic solutions of singular Rayleigh equations

$$
x^{\prime \prime}+f\left(t, x^{\prime}\right)+g(x)=p(t)
$$

where $g:(0,+\infty) \rightarrow \mathbf{R}$ is continuous and has a singularity at the origin, $f: \mathbf{R}^{2} \rightarrow \mathbf{R}^{\mathbf{2}}$ is continuous and $2 \pi$ periodic with respect to the first variable $t, p: \mathbf{R} \rightarrow \mathbf{R}$ is continuous and $2 \pi$ periodic.

Equation (1.1) can be used to model the oscillations of a clarinet reed [1]. The dynamic behaviors of (1.1) have been widely investigated due to their applications in many fields such as physics, mechanics, and the engineering technique fields (see [2-8] and the references therein). Recently, the periodic problem of equations with singularities has been studied widely because of their background in applied sciences (see [9-13] and the references therein).

When $f \equiv 0,(1.1)$ is a conservation system

$$
x^{\prime \prime}+g(x)=p(t)
$$

Assume that $g$ satisfies

$$
\text { (h) } \lim _{x \rightarrow 0^{+}} g(x)=-\infty
$$

and

$$
\frac{n^{2}}{4}<\liminf _{x \rightarrow+\infty} \frac{g(x)}{x} \leq \limsup _{x \rightarrow+\infty} \frac{g(x)}{x}<\frac{(n+1)^{2}}{4} ;
$$

(c) 2015 Wang and Ma. This article is distributed under the terms of the Creative Commons Attribution 4.0 International License (http://creativecommons.org/licenses/by/4.0/), which permits unrestricted use, distribution, and reproduction in any medium, provided you give appropriate credit to the original author(s) and the source, provide a link to the Creative Commons license, and indicate if changes were made. 
moreover, the primitive function $G$ of $g$ satisfies

$$
\left(\mathrm{h}_{2}\right) \lim _{x \rightarrow 0^{+}} G(x)=+\infty
$$

where $G(x)=\int_{1}^{x} g(u) d u$. It was proved in [10] that (1.2) has at least one $2 \pi$ periodic solution.

It is well known that time maps play an important role in studying the existence and multiplicity of periodic solutions of $(1.2)[14,15]$. Assume that $g$ satisfies

$$
\text { (h) } \lim _{x \rightarrow+\infty} g(x)=+\infty .
$$

Condition $\left(\mathrm{h}_{3}\right)$ implies that there exists a constant $d>0$ such that

$$
g(x)>\sup \{|f(t, 0)|+|p(t)|: t \in[0,2 \pi]\}, \quad \text { for } x \geq d .
$$

Let us consider the autonomous system

$$
x^{\prime \prime}+g(x)=0
$$

or its equivalent system

$$
x^{\prime}=y, \quad y^{\prime}=-g(x) .
$$

The first integral of (1.4) is the curve

$$
\Gamma_{c}: \frac{1}{2} y^{2}+G(x)=G(c)
$$

where $c$ is an arbitrary constant. From conditions $\left(\mathrm{h}_{i}\right)(i=1,2,3)$ we know that, for $c>0$ sufficiently large, $\Gamma_{c}$ is a closed curve. Let $(x(t), y(t))$ be any solution of (1.4) whose orbit is $\Gamma_{c}$. Clearly, this solution is periodic. Let $T(c)$ denote the least positive period of this solution. It is not hard to calculate

$$
T(c)=\sqrt{2} \int_{d(c)}^{c} \frac{d x}{\sqrt{G(c)-G(x)}}
$$

where $0<d(c)<c, G(d(c))=G(c), \lim _{c \rightarrow+\infty} d(c)=0$. From [10] we know that, if conditions $\left(\mathrm{h}_{i}\right)(i=1,2,3)$ hold, then

$$
\lim _{c \rightarrow+\infty} \int_{d(c)}^{1} \frac{d x}{\sqrt{G(c)-G(x)}}=0 .
$$

Now, let us set

$$
\tau(c)=\sqrt{2} \int_{1}^{c} \frac{d x}{\sqrt{G(c)-G(x)}} .
$$

In this paper, we deal with the existence of periodic solutions of (1.1) by using the asymptotic properties of the time map $\tau$. Assume that the limit 
$\left(\mathrm{h}_{4}\right) \lim _{|x| \rightarrow+\infty} \frac{f(t, x)}{x}=0$

holds uniformly for $t \in[0,2 \pi]$. We obtain the following result.

Theorem 1.1 Assume that conditions $\left(\mathrm{h}_{i}\right)(i=1,2,3,4)$ hold. Then (1.1) possesses at least one $2 \pi$ periodic solution provided that the inequality

$$
\limsup _{c \rightarrow+\infty} \tau(c)>2 \pi
$$

holds.

Using Theorem 1.1, we can obtain the following corollary.

Corollary 1.2 Assume that conditions $\left(\mathrm{h}_{i}\right)(i=1,2,3,4)$ hold. Then $(1.1)$ possesses at least one $2 \pi$ periodic solution provided that the inequality

$$
\liminf _{x \rightarrow+\infty} \frac{2 G(x)}{x^{2}}<\frac{1}{4}
$$

holds.

Throughout this paper, we always use the notations:

$$
\begin{aligned}
& \|x\|_{\infty}=\max \{|x(t)|: t \in[0,2 \pi]\}, \quad\|x\|_{1}=\int_{0}^{2 \pi}|x(t)| d t, \\
& \|x\|_{2}=\left(\int_{0}^{2 \pi} x^{2}(t) d t\right)^{\frac{1}{2}}
\end{aligned}
$$

for any continuous $2 \pi$ periodic function $x(t)$. For a function $I(c, \cdot)$, the notation $I=o(1)$ means that, for $c \rightarrow+\infty, I \rightarrow 0$ holds uniformly with respect to the other variables.

\section{A continuation lemma}

It is well known that the continuation theorem plays a key role in studying the existence of periodic solutions of ordinary differential equations. Now we shall introduce a continuation lemma for (1.1). To this end, we consider the equivalent system of (1.1),

$$
x^{\prime}=y, \quad y^{\prime}=-(g(x)+f(t, y)-p(t)) .
$$

Now, we embed system (2.1) into a family of equations with one parameter $\lambda \in[0,1]$,

$$
x^{\prime}=\lambda y, \quad y^{\prime}=-\lambda(g(x)+f(t, \lambda y)-p(t)) .
$$

Lemma 2.1 Assume that conditions $\left(\mathrm{h}_{i}\right)(i=1,2,3,4)$ hold. Suppose that there exists a constant $\zeta \geq d$ (d is given in (1.3)) such that, if $(x(t), y(t))$ is a $2 \pi$-periodic solution of system (2.2) for some $\lambda \in(0,1)$, then

$$
\max \{x(t): t \in[0,2 \pi]\} \neq \zeta, \quad t \in[0,2 \pi] .
$$

Then system (2.1) has at least one $2 \pi$-periodic solution. 
We shall use a classical consequence of Mawhin's continuation theorem [16], Theorem 7.2 to prove Lemma 2.1. For the reader's convenience, we restate it here.

Lemma 2.2 Let $\Psi=\Psi(t, z ; \lambda):[0,2 \pi] \times \mathbf{R}^{m} \times[0,1] \rightarrow \mathbf{R}^{m}$ be a continuous function and let $\Omega \subset \mathbf{R}^{m}$ be a (non-empty) open bounded set (with boundary $\partial \Omega$ and closure $\bar{\Omega}$ ). Assume the following conditions:

(1) for any $2 \pi$-periodic solution $z(t)$ of $z^{\prime}=\lambda \Psi(t, z ; \lambda)$ with $\lambda \in(0,1)$, such that $z(t) \in \bar{\Omega}$, for all $t \in[0,2 \pi]$, it follows that $z(t) \in \Omega$, for all $t \in[0,2 \pi]$;

(2) $\Psi_{0}(z) \neq 0$, for each $z \in \partial \Omega$ and $d_{B}\left(\Psi_{0}, \Omega, 0\right) \neq 0$, where

$$
\Psi_{0}(z)=\frac{1}{2 \pi} \int_{0}^{2 \pi} \Psi(t, z ; 0) d t, \quad \text { for } z \in \mathbf{R}^{m} .
$$

Then the equation $z^{\prime}=\Psi(t, z ; 1)$ has at least one $2 \pi$-periodic solution and $z(t) \in \bar{\Omega}$, for all $t \in[0,2 \pi]$.

Proof of Lemma 2.1 We shall use Lemma 2.2 to prove this continuation lemma. Set

$$
\bar{f}=\frac{1}{2 \pi} \int_{0}^{2 \pi} f(t, 0) d t, \quad \bar{p}=\frac{1}{2 \pi} \int_{0}^{2 \pi} p(t) d t .
$$

Then there exists $\tilde{t} \in[0,2 \pi]$ such that

$$
\bar{f}-\bar{p}=\frac{1}{2 \pi} \int_{0}^{2 \pi}(f(t, 0)-p(t)) d t=f(\tilde{t}, 0)-p(\tilde{t}) .
$$

From condition $\left(\mathrm{h}_{1}\right)$ we know that there exists a constant $0<d_{0}<d$ such that

$$
g(x)<-\sup \{|f(t, 0)|+|p(t)|: t \in \mathbf{R}\}, \quad 0<x \leq d_{0} .
$$

Therefore, we have

$$
g(x)<-(|f(\tilde{t}, 0)|+|p(\tilde{t})|) \leq-|f(\tilde{t}, 0)-p(\tilde{t})|=-|\bar{f}-\bar{p}|, \quad 0<x \leq d_{0} .
$$

Meanwhile, we have

$$
g(x)>|f(\tilde{t}, 0)|+|p(\tilde{t})| \geq|f(\tilde{t}, 0)-p(\tilde{t})|=|\bar{f}-\bar{p}|, \quad x \geq d .
$$

We claim that there exist constants $0<\varepsilon<d_{0}$ and $c>0$ such that, if $(x(t), y(t))$ is a $2 \pi$ periodic solution of (2.2) with $x(t) \leq \zeta, t \in[0,2 \pi]$, then

$$
\varepsilon<x(t)<\zeta, \quad-c \leq y(t) \leq c, \quad t \in[0,2 \pi] .
$$

Integrating the second equality of (2.2) on $[0,2 \pi]$ and applying the first equality of (2.2), we get

$$
\int_{0}^{2 \pi} g(x(t)) d t=-\int_{0}^{2 \pi} f\left(t, x^{\prime}(t)\right) d t+\int_{0}^{2 \pi} p(t) d t .
$$


Then we obtain

$$
-\int_{I_{1}} g(x(t)) d t=\int_{I_{2}} g(x(t)) d t+\int_{0}^{2 \pi} f\left(t, x^{\prime}(t)\right) d t-\int_{0}^{2 \pi} p(t) d t
$$

where $I_{1}=\left\{t \in[0,2 \pi]: 0<x(t)<d_{0}\right\}, I_{2}=\left\{t \in[0,2 \pi]: d_{0} \leq x(t) \leq \zeta\right\}$. Hence, we have

$$
\begin{aligned}
\int_{0}^{2 \pi}|g(x(t))| d t & =-\int_{I_{1}} g(x(t)) d t+\int_{I_{2}}|g(x(t))| d t \\
& \leq 2 \int_{I_{2}}|g(x(t))| d t+\int_{0}^{2 \pi}\left|f\left(t, x^{\prime}(t)\right)\right| d t+\int_{0}^{2 \pi}|p(t)| d t \\
& \leq 2 M_{1}+\int_{0}^{2 \pi}\left|f\left(t, x^{\prime}(t)\right)\right| d t+\|p\|_{1},
\end{aligned}
$$

where $M_{1}=2 \pi \cdot \max \left\{|g(x)|: d_{0} \leq x \leq \zeta\right\}$.

Let us take a fixed constant $\delta$ satisfying $0<\left(1+\frac{\pi}{\sqrt{3}}\right) \delta<1$. From $\left(\mathrm{h}_{4}\right)$ we see that there exists $R_{\delta}>0$ such that, for any $|s| \geq R_{\delta}$ and $t \in[0,2 \pi]$,

$$
|f(t, s)| \leq \delta|s|
$$

Set

$$
M_{2}=\max \left\{|f(t, s)|: t \in[0,2 \pi],|s| \leq R_{\delta}\right\} .
$$

Then we see that, for any $(t, s) \in \mathbf{R}^{2}$,

$$
|f(t, s)| \leq \delta|s|+M_{2}
$$

From (2.3) and (2.4) we get

$$
\int_{0}^{2 \pi}|g(x(t))| d t \leq 2 M_{1}+\delta \int_{0}^{2 \pi}\left|x^{\prime}(t)\right| d t+2 \pi M_{2}+\|p\|_{1} .
$$

Set $M=2 M_{1}+2 \pi M_{2}+\|p\|_{1}$. Then we obtain

$$
\int_{0}^{2 \pi}|g(x(t))| d t \leq \delta \sqrt{2 \pi}\left\|x^{\prime}\right\|_{2}+M
$$

From (2.2) we know that $x(t)$ satisfies the equation as follows:

$$
x^{\prime \prime}(t)+\lambda^{2}\left(f\left(t, x^{\prime}(t)\right)+g(x(t))-p(t)\right)=0 .
$$

Multiplying (2.6) by $x(t)-\bar{x}$ with $\bar{x}=\frac{1}{2 \pi} \int_{0}^{2 \pi} x(s) d s$, and integrating the equality on $[0,2 \pi]$, we get from (2.5) and (2.6)

$$
\begin{aligned}
\int_{0}^{2 \pi}\left|x^{\prime}(t)\right|^{2} d t= & \lambda^{2} \int_{0}^{2 \pi} f\left(t, x^{\prime}(t)\right)(x(t)-\bar{x}) d t+\lambda^{2} \int_{0}^{2 \pi} g(x(t))(x(t)-\bar{x}) d t \\
& -\lambda^{2} \int_{0}^{2 \pi} p(t)(x(t)-\bar{x}) d t
\end{aligned}
$$




$$
\begin{aligned}
\leq & \int_{0}^{2 \pi}\left|f\left(t, x^{\prime}(t)\right)(x(t)-\bar{x})\right| d t+\int_{0}^{2 \pi}|g(x(t))(x(t)-\bar{x})| d t \\
& +\int_{0}^{2 \pi}|p(t)(x(t)-\bar{x})| d t \\
\leq & \delta \int_{0}^{2 \pi}\left|x^{\prime}(t)\right||x(t)-\bar{x}| d t+M_{2} \int_{0}^{2 \pi}|x(t)-\bar{x}| d t \\
& +\|x-\bar{x}\|_{\infty} \int_{0}^{2 \pi}(|g(x(t))|+|p(t)|) d t \\
\leq & \left(\delta\left\|x^{\prime}\right\|_{2}+M_{2} \sqrt{2 \pi}\right)\|x-\bar{x}\|_{2}+\left(\delta \sqrt{2 \pi}\left\|x^{\prime}\right\|_{2}+M+\|p\|_{1}\right)\|x-\bar{x}\|_{\infty} .
\end{aligned}
$$

Using the Wirtinger inequality and the Sobolev inequality, we have

$$
\|x-\bar{x}\|_{2} \leq\left\|x^{\prime}\right\|_{2}, \quad\|x-\bar{x}\|_{\infty} \leq \sqrt{\frac{\pi}{6}}\left\|x^{\prime}\right\|_{2} .
$$

Then we get

$$
\int_{0}^{2 \pi}\left|x^{\prime}(t)\right|^{2} d t \leq \delta\left(1+\frac{\pi}{\sqrt{3}}\right)\left\|x^{\prime}\right\|_{2}^{2}+\left(M_{2} \sqrt{2 \pi}+\sqrt{\frac{\pi}{6}}\left(M+\|p\|_{1}\right)\right)\left\|x^{\prime}\right\|_{2},
$$

which means that

$$
\left\|x^{\prime}\right\|_{2} \leq \gamma\left\|x^{\prime}\right\|_{2}+c_{0}
$$

where $\gamma=\delta\left(1+\frac{\pi}{\sqrt{3}}\right), c_{0}=M_{2} \sqrt{2 \pi}+\sqrt{\frac{\pi}{6}}\left(M+\|p\|_{1}\right)$. Since $0<\gamma<1$, we have

$$
\left\|x^{\prime}\right\|_{2} \leq \frac{c_{0}}{1-\gamma}:=c_{1}
$$

Integrating the first equation of $(2.2)$ on $[0,2 \pi]$ and noticing $\lambda \in(0,1]$, we get

$$
\int_{0}^{2 \pi} y(t) d t=0
$$

which implies that there exists $t_{0} \in[0,2 \pi]$ such that $y\left(t_{0}\right)=0$. Then we get from (2.4), (2.5), and (2.7)

$$
\begin{aligned}
|y(t)| & \leq\left|y\left(t_{0}\right)\right|+\int_{0}^{2 \pi}\left|y^{\prime}(t)\right| d t \leq \int_{0}^{2 \pi}|g(x(t))| d t+\int_{0}^{2 \pi}\left|f\left(t, x^{\prime}(t)\right)\right| d t+\int_{0}^{2 \pi}|p(t)| d t \\
& \leq 2 \sqrt{2 \pi} \delta c_{1}+M+2 \pi M_{2}+\|p\|_{1}:=c .
\end{aligned}
$$

Therefore,

$$
\|y\|_{\infty} \leq c
$$

Let $x\left(t_{*}\right)\left(t_{*} \in[0,2 \pi]\right)$ be the minimum of $x(t)$. Then we have $x^{\prime}\left(t_{*}\right)=0$ and $x^{\prime \prime}\left(t_{*}\right) \geq 0$. Since $x\left(t_{*}\right)$ satisfies

$$
x^{\prime \prime}\left(t_{*}\right)+\lambda^{2}\left(f\left(t_{*}, 0\right)+g\left(x\left(t_{*}\right)\right)-p\left(t_{*}\right)\right)=0,
$$


we have

$$
f\left(t_{*}, 0\right)+g\left(x\left(t_{*}\right)\right) \leq p\left(t_{*}\right)
$$

Hence,

$$
g\left(x\left(t_{*}\right)\right) \leq-f\left(t_{*}, 0\right)+p\left(t_{*}\right) \leq\left|f\left(t_{*}, 0\right)\right|+\left|p\left(t_{*}\right)\right| \leq \sup \{|f(t, 0)|+|p(t)|: t \in \mathbf{R}\},
$$

which implies

$$
x\left(t_{*}\right)<d \text {. }
$$

Let $x\left(t^{*}\right)\left(t^{*} \in[0,2 \pi]\right)$ be the maximum of $x(t)$. Then we have $x^{\prime}\left(t^{*}\right)=0$ and $x^{\prime \prime}\left(t^{*}\right) \leq 0$. Similarly, we can obtain

$$
x\left(t^{*}\right)>d_{0} .
$$

From (2.9) and (2.10) we see that there exists $\bar{t} \in[0,2 \pi]$ such that

$$
d_{0} \leq x(\bar{t}) \leq d
$$

In what follows, we shall prove that there exists $0<\varepsilon<d_{0}$ such that, for any $2 \pi$ periodic solution $(x(t), y(t))$ of $(2.2)$ with $x(t) \leq \zeta, t \in[0,2 \pi]$,

$$
\varepsilon<x(t)<\zeta, \quad t \in[0,2 \pi] .
$$

The right inequality $x(t)<\zeta(t \in[0,2 \pi])$ follows directly from the condition $\max \{x(t)$ : $t \in[0,2 \pi]\} \neq \zeta$ and $x(t) \leq \zeta, t \in[0,2 \pi]$. Next, we prove the left inequality. Otherwise, there exist a sequence $\left\{\lambda_{n}\right\}$ with $\lambda_{n} \in(0,1]$ and a sequence of $2 \pi$ periodic solutions of (2.2) $\left\{\left(x_{n}(t), y_{n}(t)\right)\right\}$ (with $\lambda=\lambda_{n}$ in (2.2)), satisfying $x_{n}(t) \leq \zeta, t \in[0,2 \pi]$, and

$$
\min _{t \in[0,2 \pi]} x_{n}(t) \rightarrow 0, \quad n \rightarrow \infty
$$

Without loss of generality, we assume that, for every $n$,

$$
\min _{t \in[0,2 \pi]} x_{n}(t)<d_{0}
$$

Set $\varepsilon_{n}=x_{n}\left(t_{n}\right)=\min _{t \in[0,2 \pi]} x_{n}(t), t_{n} \in[0,2 \pi]$. From (2.11) and (2.12) we see that there exists $\alpha_{n} \in\left(t_{n}, t_{n}+2 \pi\right)$ such that

$$
x\left(\alpha_{n}\right)=d_{0}, \quad \varepsilon_{n}<x_{n}(t)<d_{0}, \quad t \in\left(t_{n}, \alpha_{n}\right) .
$$

Since $\left(x_{n}(t), y_{n}(t)\right)$ satisfies the equation

$$
y_{n}^{\prime}(t)=-\lambda_{n}\left(g\left(x_{n}(t)\right)+f\left(t, \lambda_{n} y_{n}(t)\right)-p(t)\right),
$$


we have

$$
y_{n}(t) y_{n}^{\prime}(t)=-\lambda_{n} y_{n}(t)\left(g\left(x_{n}(t)\right)+f\left(t, \lambda_{n} y_{n}(t)\right)-p(t)\right) .
$$

Recalling $x_{n}^{\prime}(t)=\lambda_{n} y_{n}(t)$, we get

$$
y_{n}(t) y_{n}^{\prime}(t)=-\left(g\left(x_{n}(t)\right)+f\left(t, x_{n}^{\prime}(t)\right)-p(t)\right) x_{n}^{\prime}(t) .
$$

Integrating both sides of (2.13) over the interval $\left[t_{n}, \alpha_{n}\right]$ and using the fact $x_{n}^{\prime}\left(t_{n}\right)=$ $\lambda_{n} y_{n}\left(t_{n}\right)=0$, we obtain

$$
\frac{1}{2} y_{n}^{2}\left(\alpha_{n}\right)=-\int_{\varepsilon_{n}}^{d_{1}} g(s) d s-\int_{t_{n}}^{\alpha_{n}} f\left(t, x_{n}^{\prime}(t)\right) x_{n}^{\prime}(t) d t+\int_{t_{n}}^{\alpha_{n}} p(t) x_{n}^{\prime}(t) d t
$$

Therefore, we get

$$
\begin{aligned}
-\int_{\varepsilon_{n}}^{d_{1}} g(s) d s= & \left|\int_{\varepsilon_{n}}^{d_{1}} g(s) d s\right| \leq \frac{1}{2} y_{n}^{2}\left(\alpha_{n}\right)+\int_{0}^{2 \pi}\left|f\left(t, x_{n}^{\prime}(t)\right) x_{n}^{\prime}(t)\right| d t \\
& +\int_{0}^{2 \pi}\left|p(t) x_{n}^{\prime}(t)\right| d t .
\end{aligned}
$$

From $\left(\mathrm{h}_{2}\right)$ we have

$$
\lim _{n \rightarrow \infty} \int_{\varepsilon_{n}}^{d_{1}} g(s) d s=-\infty
$$

Next, we shall estimate the right hand side of (2.14). First, it follows from (2.8) that we have

$$
y_{n}^{2}\left(\alpha_{n}\right) \leq c^{2}
$$

Meanwhile, according to (2.4) and (2.7), we get

$$
\begin{aligned}
\int_{0}^{2 \pi}\left|f\left(t, x_{n}^{\prime}(t)\right) x_{n}^{\prime}(t)\right| d t & \leq \delta \int_{0}^{2 \pi} x_{n}^{\prime 2}(t) d t+M_{2} \int_{0}^{2 \pi}\left|x_{n}^{\prime}(t)\right| d t \\
& \leq \delta\left\|x_{n}^{\prime}\right\|_{2}^{2}+\sqrt{2 \pi} M_{2}\left\|x_{n}^{\prime}\right\|_{2} \\
& \leq \delta c_{1}^{2}+\sqrt{2 \pi} M_{2} c_{1} .
\end{aligned}
$$

Obviously, we have

$$
\int_{0}^{2 \pi}\left|p(t) x_{n}^{\prime}(t)\right| d t \leq \sqrt{2 \pi}\|p\|_{\infty}\left\|x_{n}^{\prime}\right\|_{2} \leq \sqrt{2 \pi}\|p\|_{\infty} c_{1} .
$$

Hence, the right hand side of (2.14) is bounded. This conclusion contradicts (2.15).

To use Lemma 2.2, we define an open bounded set $\Omega=\{(x, y): \varepsilon<x<\zeta,-c-1<y<c+1\}$, and a map $S:(0,+\infty) \times \mathbf{R} \rightarrow \mathbf{R}^{2}, S(x, y)=(y,-g(x)-\bar{f}+\bar{p})$. Then, for any $2 \pi$-periodic solution $(x(t), y(t))$ of system (2.2), such that $(x(t), y(t)) \in \bar{\Omega}$, for all $t \in[0,2 \pi]$, we have $(x(t), y(t)) \in \Omega$, for all $t \in[0,2 \pi]$. Therefore, the first condition of Lemma 2.2 is satisfied. 
Obviously, $S$ does not vanish outside the rectangle $\Omega$. Furthermore, the Brouwer degree of $S, d_{B}(S, \Omega, 0)$, is defined and $d_{B}(S, \Omega, 0)=d_{B}(g,(\varepsilon, \zeta), \bar{p}-\bar{f})=1$ because $g$ is continuous and $g(\varepsilon)<\bar{p}-\bar{f}, g(\zeta)>\bar{p}-\bar{f}$. According to Lemma 2.2, system (2.1) has at least one $2 \pi$ periodic solution.

Lemma 2.3 [14] Assume that $g: \mathbf{R} \rightarrow \mathbf{R}$ is continuous and $\lim _{|x| \rightarrow+\infty} \operatorname{sgn}(x) g(x)=+\infty$. Then, for any constant $v \in \mathbf{R}$,

$$
\lim _{c \rightarrow \pm \infty} \frac{\tau_{g}(\nu, c)}{\tau_{g}(c)}=1
$$

where

$$
\tau_{g}(c)=2\left|\int_{0}^{c} \frac{d x}{\sqrt{2(\tilde{G}(c)-\tilde{G}(x))}}\right|, \quad \tau_{g}(\nu, c)=2\left|\int_{0}^{c} \frac{d x}{\sqrt{2(\tilde{G}(c)-\tilde{G}(x)+v(c-x))}}\right|
$$

with $\tilde{G}(x)=\int_{0}^{x} g(s) d s$.

Remark 2.4 When $g:[0,+\infty) \rightarrow \mathbf{R}$ is continuous and satisfies $\lim _{x \rightarrow+\infty} g(x)=+\infty$, we can also define $\tau_{g}(c)$ and $\tau_{g}(v, c)$ for $c>0$ large enough. In this case, we know from Lemma 2.3 that, for any constant $v$,

$$
\lim _{c \rightarrow+\infty} \frac{\tau_{g}(\nu, c)}{\tau_{g}(c)}=1 .
$$

When $g:(0,+\infty) \rightarrow \mathbf{R}$ is continuous and $\lim _{x \rightarrow+\infty} g(x)=+\infty$, we can get a similar estimate. Under this condition, it is noted that $g$ may have a singularity at the origin, $x=0$, namely, $\lim _{x \rightarrow 0^{+}} g(x)=-\infty$. For any constant $v \in \mathbf{R}$ and sufficiently large $c \geq 1$, let us set

$$
\tau_{g}^{+}(v, c)=2 \int_{1}^{c} \frac{d x}{\sqrt{2(G(c)-G(x)+v(c-x))}}
$$

where $G(x)=\int_{1}^{x} g(s) d s$. Then we have

$$
\lim _{c \rightarrow+\infty} \frac{\tau_{g}^{+}(v, c)}{\tau(c)}=1
$$

where $\tau$ is defined by (1.5).

In fact, let us consider a function $g_{0}:[0,+\infty) \rightarrow \mathbf{R}, g_{0}(x)=g(x+1), x \geq 0$. Obviously, $g_{0}$ is continuous on the interval $[0,+\infty)$ and satisfies $\lim _{x \rightarrow+\infty} g_{0}(x)=+\infty$. Then we have, for $x \geq 0$,

$$
\tilde{G}_{0}(x)=\int_{0}^{x} g_{0}(s) d s=\int_{1}^{x+1} g(s) d s=G(x+1) .
$$

According to Lemma 2.3, we get

$$
\lim _{c \rightarrow+\infty} \frac{\tau_{g_{0}}(v, c)}{\tau_{g_{0}}(c)}=1
$$


When $c>0$ is large enough, we have

$$
\begin{aligned}
\tau_{g_{0}}(v, c) & =2 \int_{0}^{c} \frac{d x}{\sqrt{2\left(\tilde{G}_{0}(c)-\tilde{G}_{0}(x)+v(c-x)\right)}} \\
& =2 \int_{0}^{c} \frac{d x}{\sqrt{2(G(c+1)-G(x+1)+v(c-x))}} \\
& =2 \int_{1}^{c+1} \frac{d x}{\sqrt{2(G(c+1)-G(x)+v(c+1-x))}}=\tau_{g}^{+}(v, c+1) .
\end{aligned}
$$

Similarly, we have

$$
\tau_{g_{0}}(c)=\tau(c+1)
$$

Consequently, we get

$$
\lim _{c \rightarrow+\infty} \frac{\tau_{g}^{+}(v, c+1)}{\tau(c+1)}=1
$$

Therefore, the conclusion (2.16) holds.

\section{Proof of Theorem 1.1}

In this section, we shall use the continuation Lemma 2.1 given in Section 2 to prove Theorem 1.1.

Proof of Theorem 1.1 Let us set

$$
\tau=\limsup _{c \rightarrow+\infty} \tau(c)>2 \pi
$$

Then there exist $0<\varepsilon_{0}<\frac{1}{3}(\tau-2 \pi)$ and a sequence $\left\{c_{n}\right\}$ with $\lim _{n \rightarrow \infty} c_{n}=+\infty$ such that, for every $n$,

$$
\tau\left(c_{n}\right)>\tau-\varepsilon_{0}>2 \pi+2 \varepsilon_{0} .
$$

We shall prove that the condition of Lemma 2.1 is satisfied for $\zeta=c_{n}$ with $n$ sufficiently large.

Let $(x(t), y(t))$ be any $2 \pi$ periodic solution of (2.2) for some $\lambda \in(0,1]$ and suppose that, for $n$ large enough,

$$
x\left(t^{*}\right)=\max _{t \in[0,2 \pi]} x(t)=c_{n}>d,
$$

where $d$ is given in (1.3). Assume that $x\left(t_{*}\right)\left(t_{*} \in[0,2 \pi]\right)$ is a local minimum of $x(t)$. From the proof of Lemma 2.1

$$
x\left(t_{*}\right)<d .
$$


Then there exists an interval $[\alpha, \beta] \subset[0,2 \pi]$ containing $t^{*}$, with $\alpha=\alpha(x, \lambda), \beta=\beta(x, \lambda)$ such that

$$
x(\alpha)=x(\beta)=d ; \quad x(t)>d, \quad t \in(\alpha, \beta)
$$

and

$$
y\left(t^{*}\right)=0 ; \quad y(t)>0, \quad t \in\left[\alpha, t^{*}\right), \quad y(t)<0, \quad t \in\left(t^{*}, \beta\right] .
$$

From (2.2) we have

$$
y(t) y^{\prime}(t)+\lambda(f(t, \lambda y(t))+g(x(t))-p(t)) y(t)=0 .
$$

Integrating both sides of (3.1) on the interval $\left[t, t^{*}\right]$ with $\alpha \leq t \leq t^{*}$, we have

$$
y^{2}(t)=2\left(G\left(x\left(t^{*}\right)\right)-G(x(t))\right)+2 \lambda \int_{t}^{t^{*}} f(\tau, \lambda y(\tau)) y(\tau) d \tau-2 \lambda \int_{t}^{t^{*}} p(\tau) y(\tau) d \tau .
$$

From $\left(\mathrm{h}_{4}\right)$ we know that, for any sufficiently small $\varepsilon>0$, there is a constant $M_{\varepsilon}>0$ such that, for any $(t, y) \in R^{2}$,

$$
|f(t, y)| \leq \varepsilon|y|+M_{\varepsilon} .
$$

Since $y(t)>0, t \in\left[\alpha, t^{*}\right]$, it follows from (3.2) and (3.3) that, for $t \in\left[\alpha, t^{*}\right]$,

$$
\begin{aligned}
y^{2}(t) \leq & 2\left(G\left(x\left(t^{*}\right)\right)-G(x(t))\right)+2 \int_{t}^{t^{*}}|f(\tau, \lambda y(\tau))||\lambda y(\tau)| d \tau+2 \int_{t}^{t^{*}}|p(\tau)||\lambda y(\tau)| d \tau \\
& \leq 2\left(G\left(x\left(t^{*}\right)\right)-G(x(t))\right)+2 \varepsilon \int_{t}^{t^{*}} y^{2}(\tau) d \tau+2 \int_{t}^{t^{*}}\left(|p(\tau)|+M_{\varepsilon}\right)|\lambda y(\tau)| d \tau \\
& \leq 2\left(G\left(x\left(t^{*}\right)\right)-G(x(t))\right)+2 \varepsilon \int_{t}^{t^{*}} y^{2}(\tau) d \tau+M_{\varepsilon}^{\prime} \int_{t}^{t^{*}} x^{\prime}(\tau) d \tau \\
& =2\left(G\left(x\left(t^{*}\right)\right)-G(x(t))\right)+2 \varepsilon \int_{t}^{t^{*}} y^{2}(\tau) d \tau+M_{\varepsilon}^{\prime}\left(x\left(t^{*}\right)-x(t)\right),
\end{aligned}
$$

where $M_{\varepsilon}^{\prime}=M_{\varepsilon}+\|p\|_{\infty}$. Let us set

$$
\phi(t)=\int_{t}^{t^{*}} y^{2}(\tau) d \tau
$$

Then we have

$$
\phi^{\prime}(t)=-y^{2}(t)
$$

Hence,

$$
-\phi^{\prime}(t)-2 \varepsilon \phi(t) \leq 2\left(G\left(x\left(t^{*}\right)\right)-G(x(t))\right)+M_{\varepsilon}^{\prime}\left(x\left(t^{*}\right)-x(t)\right) .
$$


Multiplying both sides of (3.4) by $e^{2 \varepsilon t}$ and integrating over the interval $\left[t, t^{*}\right]$ yields

$$
-\int_{t}^{t^{*}}\left[\phi(\tau) e^{2 \varepsilon \tau}\right]^{\prime} d \tau \leq \int_{t}^{t^{*}}\left[2\left(G\left(x\left(t^{*}\right)\right)-G(x(\tau))\right)+M_{\varepsilon}^{\prime}\left(x\left(t^{*}\right)-x(\tau)\right)\right] e^{2 \varepsilon \tau} d \tau .
$$

Since $\phi\left(t^{*}\right)=0$, we have

$$
\phi(t) e^{2 \varepsilon t} \leq \int_{t}^{t^{*}}\left[2\left(G\left(x\left(t^{*}\right)\right)-G(x(\tau))\right)+M_{\varepsilon}^{\prime}\left(x\left(t^{*}\right)-x(\tau)\right)\right] e^{2 \varepsilon \tau} d \tau .
$$

From $x^{\prime}(t)=\lambda y(t) \geq 0, t \in\left[\alpha, t^{*}\right]$ we know that $x(t)$ is increasing on the interval $\left[\alpha, t^{*}\right]$. Therefore, we get, for $t \in\left[\alpha, t^{*}\right]$,

$$
\phi(t) e^{2 \varepsilon t} \leq e^{2 \varepsilon t^{*}} \int_{t}^{t^{*}}\left[2\left(G\left(x\left(t^{*}\right)\right)-G(x(\tau))\right)+M_{\varepsilon}^{\prime}\left(x\left(t^{*}\right)-x(\tau)\right)\right] d \tau .
$$

Furthermore,

$$
\phi(t) \leq 2 \pi e^{4 \pi \varepsilon}\left[2\left(G\left(x\left(t^{*}\right)\right)-G(x(t))\right)+M_{\varepsilon}^{\prime}\left(x\left(t^{*}\right)-x(t)\right)\right] .
$$

Consequently, we can get, for $t \in\left[\alpha, t^{*}\right]$,

$$
y^{2}(t) \leq(1+\kappa(\varepsilon))\left[2\left(G\left(x\left(t^{*}\right)\right)-G(x(t))\right)+M_{\varepsilon}^{\prime}\left(x\left(t^{*}\right)-x(t)\right)\right],
$$

where $\kappa(\varepsilon)=4 \pi \varepsilon e^{4 \pi \varepsilon}$. Recalling $x^{\prime}(t)=\lambda y(t)$ and $y(t)>0$ for $t \in\left[\alpha, t^{*}\right]$, we have

$$
x^{\prime}(t) \leq \sqrt{1+\kappa(\varepsilon)} \sqrt{2\left(G\left(x\left(t^{*}\right)\right)-G(x(t))\right)+M_{\varepsilon}^{\prime}\left(x\left(t^{*}\right)-x(t)\right)} .
$$

Hence,

$$
\frac{x^{\prime}(t)}{\sqrt{1+\kappa(\varepsilon)} \sqrt{2\left(G\left(x\left(t^{*}\right)\right)-G(x(t))\right)+M_{\varepsilon}^{\prime}\left(x\left(t^{*}\right)-x(t)\right)}} \leq 1 .
$$

Integrating both sides of (3.5) over interval $\left[\alpha, t^{*}\right]$ yields

$$
\frac{1}{\sqrt{1+\kappa(\varepsilon)}} \int_{d_{2}}^{c_{n}} \frac{d x}{\sqrt{2\left(G\left(c_{n}\right)-G(x)\right)+M_{\varepsilon}^{\prime}\left(c_{n}-x\right)}} \leq t^{*}-\alpha .
$$

Similarly, we can get

$$
\frac{1}{\sqrt{1+\kappa(\varepsilon)}} \int_{d_{2}}^{c_{n}} \frac{d x}{\sqrt{2\left(G\left(c_{n}\right)-G(x)\right)+M_{\varepsilon}^{\prime}\left(c_{n}-x\right)}} \leq \beta-t^{*} .
$$

Therefore, we obtain

$$
\frac{2}{\sqrt{1+\kappa(\varepsilon)}} \int_{d_{2}}^{c_{n}} \frac{d x}{\sqrt{2\left(G\left(c_{n}\right)-G(x)\right)+M_{\varepsilon}^{\prime}\left(c_{n}-x\right)}} \leq \beta-\alpha .
$$

Using $\left(\mathrm{h}_{3}\right)$ we can easily derive that, for $n \rightarrow \infty$,

$$
\int_{1}^{d_{2}} \frac{d x}{\sqrt{2\left(G\left(c_{n}\right)-G(x)\right)+M_{\varepsilon}^{\prime}\left(c_{n}-x\right)}}=o(1) .
$$


Then we have

$$
\frac{2}{\sqrt{1+\kappa(\varepsilon)}} \int_{1}^{c_{n}} \frac{d x}{\sqrt{2\left(G\left(c_{n}\right)-G(x)\right)+M_{\varepsilon}^{\prime}\left(c_{n}-x\right)}}+o(1) \leq \beta-\alpha .
$$

It follows from Remark 2.4 that

$$
\lim _{n \rightarrow \infty} \frac{1}{\tau\left(c_{n}\right)} \int_{1}^{c_{n}} \frac{2 d x}{\sqrt{2\left(G\left(c_{n}\right)-G(x)\right)+M_{\varepsilon}^{\prime}\left(c_{n}-x\right)}}=1
$$

Consequently, we have

$$
\int_{1}^{c_{n}} \frac{2 d x}{\sqrt{2\left(G\left(c_{n}\right)-G(x)\right)+M_{\varepsilon}^{\prime}\left(c_{n}-x\right)}}=\tau\left(c_{n}\right)(1+o(1)) .
$$

Furthermore,

$$
\int_{1}^{c_{n}} \frac{2 d x}{\sqrt{2\left(G\left(c_{n}\right)-G(x)\right)+M_{\varepsilon}^{\prime}\left(c_{n}-x\right)}} \geq 2 \pi+2 \varepsilon_{0}+o(1) .
$$

Since $\lim _{\varepsilon \rightarrow 0^{+}} \sqrt{1+\kappa(\varepsilon)}=1$, there exist a sufficiently small $\varepsilon>0$ and a sufficiently large $n$ such that, if $\max _{[0,2 \pi]} x(t)=c_{n}$, then

$$
\beta-\alpha>2 \pi+\varepsilon_{0}
$$

which contradicts with the inequality $\beta-\alpha<2 \pi$. Then we find $\zeta=c_{n}$ for $n$ sufficiently large. Consequently, from the continuation Lemma 2.1, we know that (2.1) has at least one $2 \pi$ periodic solution.

Proof of Corollary 1.2 Let us denote $\rho=\liminf _{x \rightarrow+\infty} \frac{2 G(x)}{x^{2}}<\frac{1}{4}$. Then there exists $\varepsilon>0$ such that $\rho_{\varepsilon}=\rho+\varepsilon \in\left(\rho, \frac{1}{4}\right)$. Define

$$
\psi(x)=\rho_{\varepsilon} x^{2}-2 G(x), \quad x \geq 1 .
$$

Therefore, we have

$$
\limsup _{x \rightarrow+\infty} \psi(x)=\limsup _{x \rightarrow+\infty} x^{2}\left(\rho_{\varepsilon}-\frac{2 G(x)}{x^{2}}\right)=+\infty
$$

It follows that there exists a sequence $\left\{c_{n}\right\}$ with $\lim _{n \rightarrow+\infty} c_{n}=+\infty$ such that

$$
\psi(x) \leq \psi\left(c_{n}\right), \quad x \in\left(1, c_{n}\right) .
$$

Consequently,

$$
2\left(G\left(c_{n}\right)-G(x)\right) \leq \rho_{\varepsilon}\left(c_{n}^{2}-x^{2}\right), \quad x \in\left(1, c_{n}\right) .
$$

Hence, we have

$$
\tau\left(c_{n}\right)=2 \int_{1}^{c_{n}} \frac{d x}{\sqrt{2\left(G\left(c_{n}\right)-G(x)\right)}} \geq 2 \int_{1}^{c_{n}} \frac{d x}{\sqrt{\rho_{\varepsilon}\left(c_{n}^{2}-x^{2}\right)}}=\frac{2}{\sqrt{\rho_{\varepsilon}}}\left(\frac{\pi}{2}-\arcsin \frac{1}{c_{n}}\right) .
$$


As a result, we get

$$
\limsup _{n \rightarrow+\infty} \tau\left(c_{n}\right) \geq \frac{\pi}{\sqrt{\rho_{\varepsilon}}}>2 \pi
$$

which implies that $\limsup _{c \rightarrow+\infty} \tau(c)>2 \pi$. According to Theorem 1.1, (1.1) has at least one $2 \pi$ periodic solution.

Remark 3.1 In [12], the existence of periodic solutions of the Hamiltonian systems of the type

$$
x^{\prime}=g_{1}(t, y), \quad y^{\prime}=-g_{2}(t, x)
$$

was studied. A similar result was obtained (see [12], Corollary 3.13) for system (3.6). However, this corollary cannot be applied directly to obtain the main results of this paper because the asymptotic behavior of the primitive $G$ of the nonlinearity $g$ is treated in present paper.

Competing interests

The authors declare that they have no competing interests.

\section{Authors' contributions}

ZW proved a continuation lemma for Rayleigh equations. TM participated in obtaining a prior estimate and helped to draft the manuscript. All authors read and approved the final manuscript.

\section{Author details}

${ }^{1}$ School of Mathematical Sciences, Capital Normal University, Beijing, 100048, People's Republic of China. ${ }^{2}$ Editorial Department of Journal, Capital Normal University, Beijing, 100048, People's Republic of China.

\section{Acknowledgements}

The authors are grateful to the referees for many valuable suggestions to make the paper more readable. Research supported by National Nature Science Foundation of China, No. 11501381 and the Grant of Beijing Education Committee Key Project, No. KZ201310028031.

Received: 21 March 2015 Accepted: 26 August 2015 Published online: 04 September 2015

\section{References}

1. Radhakrishnan, S: Exact solutions of Rayleigh's equation and sufficient conditions for inviscid instability of parallel, bounded shear flows. Z. Angew. Math. Phys. 45, 615-637 (1994)

2. Smith, HL: On the small oscillations of the periodic Rayleigh equation. Q. Appl. Math. 44, 223-247 (1986)

3. Smith, RA: Period bounds for generalized Rayleigh equation. Int. J. Non-Linear Mech. 6, 271-277 (1977)

4. Omari, P, Villari, G: On a continuation lemma for the study of a certain planar system with applications to Liénard and Rayleigh equations. Results Math. 14, 156-173 (1998)

5. Ma, T, Wang, Z: A continuation lemma and its applications to periodic solutions of Rayleigh differential equations with subquadratic potential conditions. J. Math. Anal. Appl. 385, 1107-1118 (2012)

6. Ma, T: Periodic solutions of Rayleigh equations via time-maps. Nonlinear Anal. 75, 4137-4144 (2012)

7. Habets, P, Torres, PJ: Some multiplicity results for periodic solutions of a Rayleigh differential equation. Dyn. Contin. Discrete Impuls. Syst., Ser. A Math. Anal. 8, 335-351 (2001)

8. Si, J: Invariant tori and unboundedness for second order differential equations with asymmetric nonlinearities depending on the derivatives. Nonlinear Anal. 67, 3098-3115 (2007)

9. Habets, P, Sanchez, L: Periodic solutions of some Liénard equations with singularities. Proc. Am. Math. Soc. 109, 1035-1044 (1990)

10. Wang, Z: Periodic solutions of the second order differential equations with singularities. Nonlinear Anal. 58, 319-331 (2004)

11. Sfecci, A: Nonresonance conditions for radial solutions of nonlinear Neumann elliptic problems on annuli. Rend. Ist. Mat. Univ. Trieste 46, 255-270 (2014)

12. Fonda, A, Sfecci, A: A general method for the existence of periodic solutions of differential systems in the plane. J. Differ. Equ. 252, 1369-1391 (2012)

13. Fonda, A, Toader, R: Periodic orbits of radially symmetric Keplerian-like systems: a topological degree approach. J. Differ. Equ. 244, 3235-3264 (2008)

14. Opial, Z: Sur les périodes des solutions de l'équation différentielle $x^{\prime \prime}+g(x)=0$. Ann. Pol. Math. 10, 49-72 (1961)

15. Fonda, A, Zanolin, F: On the use of time-maps for the solvability of nonlinear boundary value problems. Arch. Math. 59, 245-259 (1992)

16. Mawhin, J: Equivalence theorems for nonlinear operator equations and coincidence degree theory for some mappings in locally convex topological vector spaces. J. Differ. Equ. 12, 610-636 (1972) 